\title{
Effects of Some Non Steroidal Anti-inflammatory Drugs on Ovulation in Women with Mild Musculoskeletal Pain (A Clinical Study)
}

\author{
Sherif B. ${ }^{1}$, Dr.Al-Zohyri A.M ${ }^{1}$,Dr. Shihab S.S ${ }^{2}$ \\ Pharmacology, College of Medicine, Baghdad University, Baghdad, Iraq \\ Rheumatology, College of Medicine, Baghdad University, Baghdad, Iraq
}

\begin{abstract}
NSAIDs are popular drugs used for variable conditions, being analgesics, antipyretics and antiinflammatory agents for more than a century. They are sold without a prescription and taken by millions of patients every day all over the world. Since they are used on a large scale as an anti-inflammatory agents \& are given in doses that are consider high as compared to doses used in conditions other than inflammation and because many of NSAIDs users are females at child bearing age, so the present study was conducted to investigate the effects of diclofenac, naproxen \& etoricoxib on ovulation \& progesterone level in women.

Adults women (39 females at fertile age) were chosen as volunteers to take part in this study. They were visited the Rheumatology consultation clinic in Baghdad Hospital, suffering from minor aches \& were diagnosed \& received one of the three test drugs included in this study (diclofenac 100mg once daily, naproxen500mg twice daily \& etoricoxib $90 \mathrm{mg}$ once daily).

Treatment with the above drugs was lasted for ten days starting at day ten after the onset of the period, a blood sample was taken from each patients for hormonal analysis ( progesterone level) together with an ultra sonsography $(U / S)$ to assess the mean diameter of the dominant follicle. At day twenty the patient came back for another ultra sonography \& to give a blood sample for another check for progesterone level. A fourth group served as controls, who received no treatment (volunteers).

The present study presents several new findings:

1. A significant inhibition of ovulation have been observed in patients teated with diclofenac, naproxen \& etoricoxib.

2. Diclofenac was the highest inhibitor of ovulation compared to the other two drugs ( naproxen \& etoricoxib)

3. A significant decrease in progesterone level in all three groups in compared to the control group.

4. Functional cysts have been observed in one third of patients by the end of the treatment period with diclofenac, naproxen \& etoricoxib due to unruptured follicles (disappeared at the next cycle).

The above new findings have a direct implication \& importance in relation to conditions where NSAIDs ( diclofenac, naproxen \& eroricoxib) used and may serve as an alarm of the harmful effects of these drugs on female fertility \& these effects should be taken into consideration in females planning to have a child. In the other hand, the above results may open the door for looking for an emergency contraceptive tablet safer than those at use nowadays.
\end{abstract}

\section{Introduction}

Most NSAIDs act as nonselective inhibitors of the enzyme cyclooxygenase (COX), inhibiting both the cyclooxygenase-1 (COX-1) and cyclooxygenase-2 (COX-2) isoenzymes. This COX enzyme catalyzes the formation of prostaglandins (PG) and thromboxane from arachidonic acid (1).

Since PGs play a central role in inflammation, and the ovulatory process has all the signs of an acute, selfcontrolled inflammatory reaction, including hyperaemia, edema, leukocyte extravasation, and induction of proteolytic and collagenolytic activities, PGs have been recognized as key mediators of ovulation for $>30$ years, $(2,3)$, a potential relationship between prostaglandin biosynthesis and ovulation first emerged during the early 1970 s $(4,3)$

Prostaglandins $\left(\mathrm{E}_{2} \& \mathrm{~F}_{2 \alpha}\right)$ and proteolytic enzymes such as collagenase and plasmin, are increased in response to LH surge and progesterone increment prior to ovulation. Suggesting that Proteolytic enzymes and prostaglandins are activated and digest collagen in the follicular wall, leading to an explosive release of the oocyte-cumulus complex (3).

During the time when scientists continued to look for the role of PG in ovulation, a study was done to test the hypothesis that COX-2 could be used as a marker of follicular commitment to ovulation during ovarian 
stimulation, multiple follicular development was stimulated with exogenous FSH, ovulation was induced with hCG, and the pattern of COX-2 expression was characterized (5). Results of this study showed that COX-2 expression $24 \mathrm{~h}$ after hCG was detected in $76 \%$ of follicles $>8 \mathrm{~mm}$, but the proportion (24\%) of follicles not expressing the enzyme was very similar to the incidence of anovulatory follicles detected by ultrasonography .Interestingly, in contrast to COX-2-positive follicles, COX-2-negative follicles were not luteinized and contained a compact cumulus-oocyte complex, suggesting an apparent overall failure to respond to the gonadotrophin pre-ovulatory signal. Thus, COX-2 expression should provide a valuable marker to unravel the molecular basis behind the development of large anovulatory follicles during ovarian stimulation (5).

This study was designed to study the possible side effects of short term ( 10days ) use of either diclofenac, naproxen or etoricoxeb on ovulation and hormonal levels in normally cycling women.

\section{Patients, materials \& methods}

97 Iraqi females participate in this study, including 75 as patients $\& 22$ of them as controls, but the actual number of patients \& controls who continued till the end of the study were 49 , divided as following:

1. 16 of them treated with diclofenac $100 \mathrm{mg}$ daily.

2. 12 treated with naproxen $500 \mathrm{mg}$ twice daily.

3. 11 treated with etoricoxib $90 \mathrm{mg}$ daily.

4. 10 controls.

All the patients treated for 10 days period, starting the treatment in the late follicular phase (day10-12) after the onset of their menstrual cycle. Before given the treatment, patients sent for U/S that was performed to assess the mean diameter of the dominant follicle, endometrial thickness and ovarian size, also a venous blood sample was taken at the same time of U/S, then to be centrifuged at $3000 \mathrm{RPM}$ for 5 minutes to get the serum (about $1.5 \mathrm{ml}$ ) which would be transferred into a plastic tube and stored in a deep freezer at $-20 \mathrm{C}^{\circ}$. After 10 days period of treatment with either of the mentioned NSAIDS, U/S were repeated and blood samples were taken again, centrifuged and stored in the same way until used for hormonal determination.

Progesterone $\left(\mathrm{P}_{4}\right)$ level have been tested for each patient included in this study before \& after treatment with either diclofenac100mg once daily, naproxen 500mg twice daily, or etoricoxib 90mg once daily, and also for the controls, these tests have been analyzed in the High Institute For Infertility Diagnosis \& Assisted Reproductive Technologies lab, at AL-Nahrain University, using mini VIDAS technique.

Statistical analysis : Collected data were analyzed using:

- Descriptive statistic , by using tables and figures .

- Analytical statistic, by using Microsoft excel 2007 , minitab ,v 17 ,student s T test, ANOVA test, Chi test .

- $\quad$ Statistical significant $(\mathrm{P}<0.05)$.

\section{Results}

Diclofenac, naproxen \& etoricoxib exhibited a highly inhibitory effects on ovulation compared to the control, this inhibitory effect demonstrated by growing follicle without ovulation during the treatment period for the three drugs: diclofenac (22 \pm 2.5$)$, naproxen $(17.6 \pm 3.8) \&$ etoricoxib (19.8 \pm 4.4$)$ respectively, Table 1, Fig. 1.

At the same time diclofenac, naproxen \& etoricoxib caused a highly significant decrease in the mean $\mathrm{P}_{4}$ level ( $\mathrm{P}<0.01$ ), in comparison with the control during luteal phase, Table 2, Fig.2.

A non significant differences in the E.TH. between each treated group with the control $(\mathrm{P}>0.05)$, have been observed which indicate normal E.TH. after treatment with NSAIDs in the luteal phase, with the highest thickness observed with etoricoxib group.

From another point of view, low percentage of ovulation have been observed in patients treated with either of the three drugs, in comparing with controls $(100 \%)$ ovulation, while $(6.25 \%, 25 \% \& 27.3 \%)$ for diclofenac, naproxen \& etoricoxib respectively, and in turn a high percentage of reduction in ovulation especially with diclofenac ( $93.75 \%$ ), Table 3, Fig.3.

Regarding the fate of dominant follicle at the end of the treatment period ( day 20-22) with diclofenac, naproxen \& etoricoxib show :

A) Ovulation occur in $1 / 16$ of diclofenac group ( $6.25 \%), 3 / 12$ of naproxen group ( $25 \%$ ) \& $3 / 11$ of etoricoxib group ( $27.3 \%$ ).

B) Unruptured follicle occur in 12/16 of diclofenac group ( $75 \%$ ), 4/12 for naproxen group ( $33.3 \%$ ) \& $2 / 11$ for etoricoxib group ( $18.2 \%)$.

C) cyst occur in 3/16 for diclofenac group (18.75\%), 5/12 for naproxen group ( $41.7 \%) \& 6 / 11$ for etoricoxib group (54.5\%), Table 4, Fig.4. 
Table 1: Effects of Diclofenac, Naproxen \& Etoricoxib on DF during luteal phase.

\begin{tabular}{|c|c|c|c|c|c|}
\hline \multirow{2}{*}{$\begin{array}{c}\text { Dominant follicle } \\
\text { diameter(mm) }\end{array}$} & \multicolumn{2}{|c|}{ control } & \multicolumn{2}{c|}{ Treated groups } & \multirow{2}{*}{ * $^{*}$} \\
\cline { 2 - 6 } & mean & $\pm S E$ & mean & $\pm S E$ & Nop \\
\hline Diclofenac & 0 & 0 & 22 & 2.5 & Nop \\
\hline Naproxen & 0 & 0 & 17.6 & 3.8 & \\
\hline Etoricoxib & 0 & 0 & 19.8 & 4.4 & Nop \\
\hline
\end{tabular}

Values are mean \pm standard error ( SE), $(\mathrm{n}=16$ diclofenac, 12 naproxen , 11 etoricoxib \& 10 controls). $\mathrm{P}^{*}=$ statistical level of significant. $\mathrm{T}$ test.

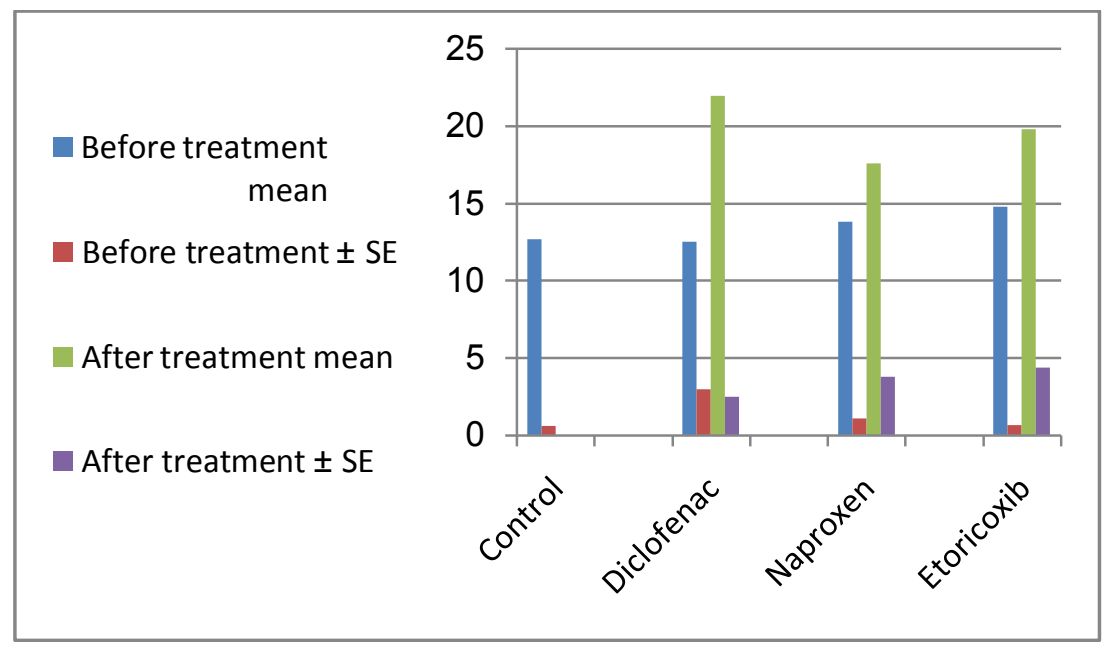

Fig 1: Effects of Diclofenac, Naproxen \& Etoricoxib on DF in human females ( before \& after treatment).

Table 2: Effects of Diclofenac, Naproxen \& Etoricoxib on progesterone level $\left(\mathrm{P}_{4}\right)$ during luteal phase:

\begin{tabular}{|c|c|c|c|c|c|}
\hline \multirow{2}{*}{$\begin{array}{c}\text { Progesterone level } \\
(n g / m l)\end{array}$} & \multicolumn{2}{|c|}{ control } & \multicolumn{2}{|c|}{ Treated groups } & \multirow[t]{2}{*}{$p$} \\
\hline & mean & $\pm S E$ & mean & $\pm S E$ & \\
\hline & 15.9 & 0.8 & 6.62 & 1.2 & $0.001 *$ \\
\hline \multicolumn{6}{|l|}{ Diclofenac } \\
\hline & 15.9 & 0.8 & 7.37 & 1.5 & $0.003 *$ \\
\hline \multicolumn{6}{|l|}{ Naproxen } \\
\hline Etoricoxib & 15.9 & 0.8 & 11.5 & 1.5 & $0.027 a$ \\
\hline
\end{tabular}

Values are mean \pm standard error ( SE), ( $\mathrm{n}=16$ diclofenac, 12 naproxen , 11 etoricoxib \& 10 controls). ${ }^{*}=$ highly significant differences. $\mathrm{Q}=$ significant differences. $\mathrm{T}$ test. 


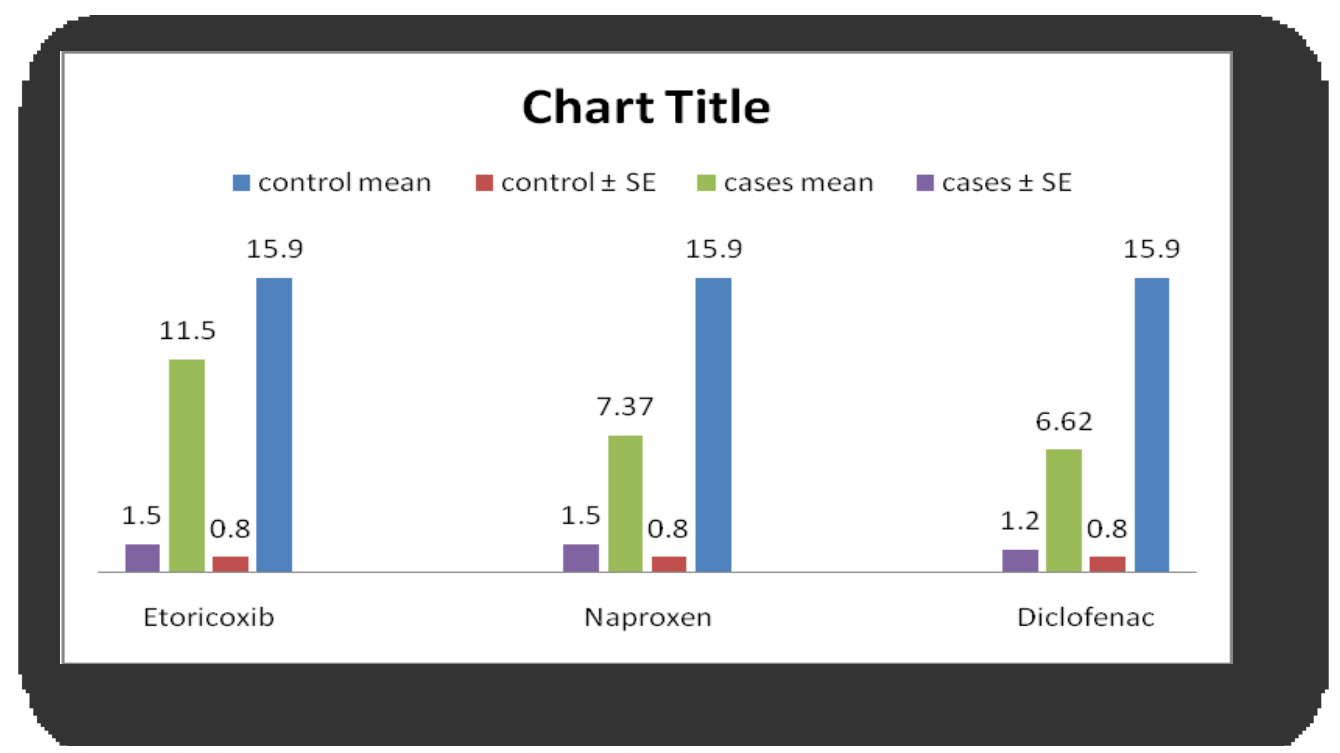

Fig.2: Effects of Diclofenac, Naproxen \& Etoricoxib on progesterone level $\left(\mathrm{P}_{4}\right)$ during luteal phase.

Table 3: Reduction in ovulation by diclofenac, naproxen \& etoricoxeb:

\begin{tabular}{|l|l|l|}
\hline Drugs/control & $\%$ Ovulation after Treatment & $\%$ reduction in ovulation after Treatment \\
\hline Control & $100 \%$ & $0 \%$ \\
\hline Diclofenac & $6.25 \%$ & $93.75 \%$ \\
\hline Naproxen & $25 \%$ & $75 \%$ \\
\hline Etoricoxib & $27.30 \%$ & $72.70 \%$ \\
\hline
\end{tabular}

Fig3: Percentage reduction in ovulation by diclofenac, naproxen \& etoricoxib.

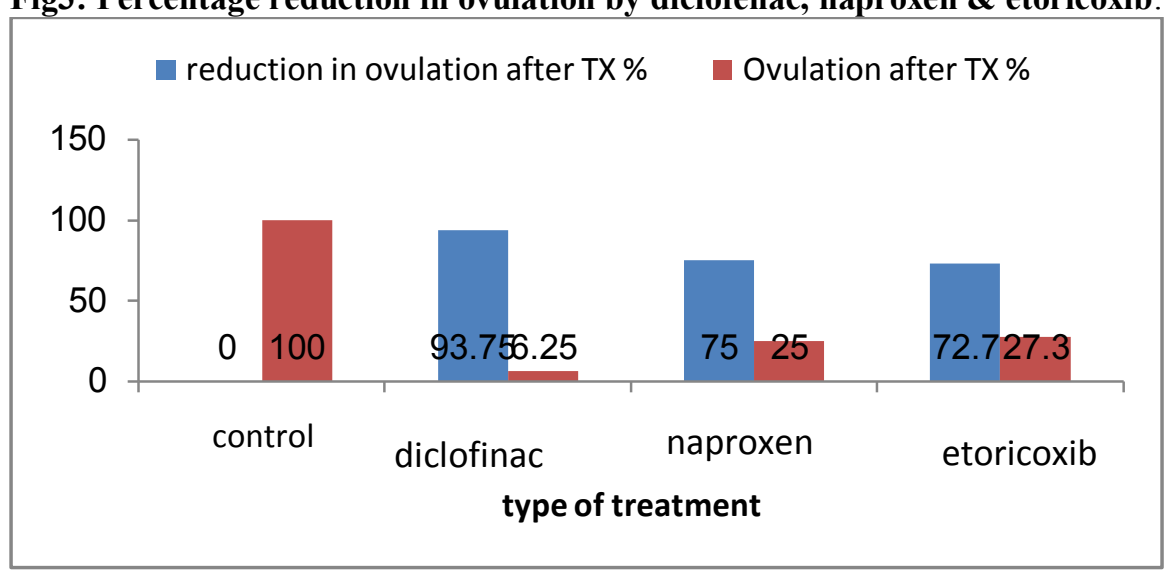

Table 4: Fate of dominant follicle at the end of the treatment period ( day 20-22) with diclofenac, naproxen \& etoricoxib:

\begin{tabular}{|c|c|c|c|c|c|c|}
\hline Day 20-22 & DICLOFINAC & NAPROXEN & ETORICOXIB & CONTROL & $\begin{array}{c}\text { Total- } \\
\text { treated } \\
\text { cases }\end{array}$ & $P$ \\
\hline Ovulation & $(6.25 \%)$ & $\begin{array}{c}3 \\
(25 \%)\end{array}$ & $\begin{array}{c}3 \\
(27.3 \%)\end{array}$ & $\begin{array}{c}10 \\
(100 \%)\end{array}$ & 7 & \multirow{2}{*}{$0.045^{*}$} \\
\hline $\begin{array}{c}\text { Un ruptured } \\
\text { Follicle }\end{array}$ & $\begin{array}{c}12 \\
(75 \%)\end{array}$ & $\begin{array}{c}4 \\
(18.3 \%)\end{array}$ & 0 & 18 \\
\hline Cyst & $\begin{array}{c}3 \\
(18.75 \%)\end{array}$ & $\begin{array}{c}5 \\
(41.7 \%)\end{array}$ & $\begin{array}{c}6 \\
(54.5 \%)\end{array}$ & 0 & 14 & 39 \\
\hline total & 16 & 12 & 11 & 10 & 39 \\
\hline
\end{tabular}

The values are numbers of patients \& $\%$ proportion of DF fate to the total number,$(n=16$ diclofenac, 12 naproxen, 11 etoricoxib \& 10 controls $). *$ significant differences $(\mathrm{P}<0.05)$.Chi square test. 


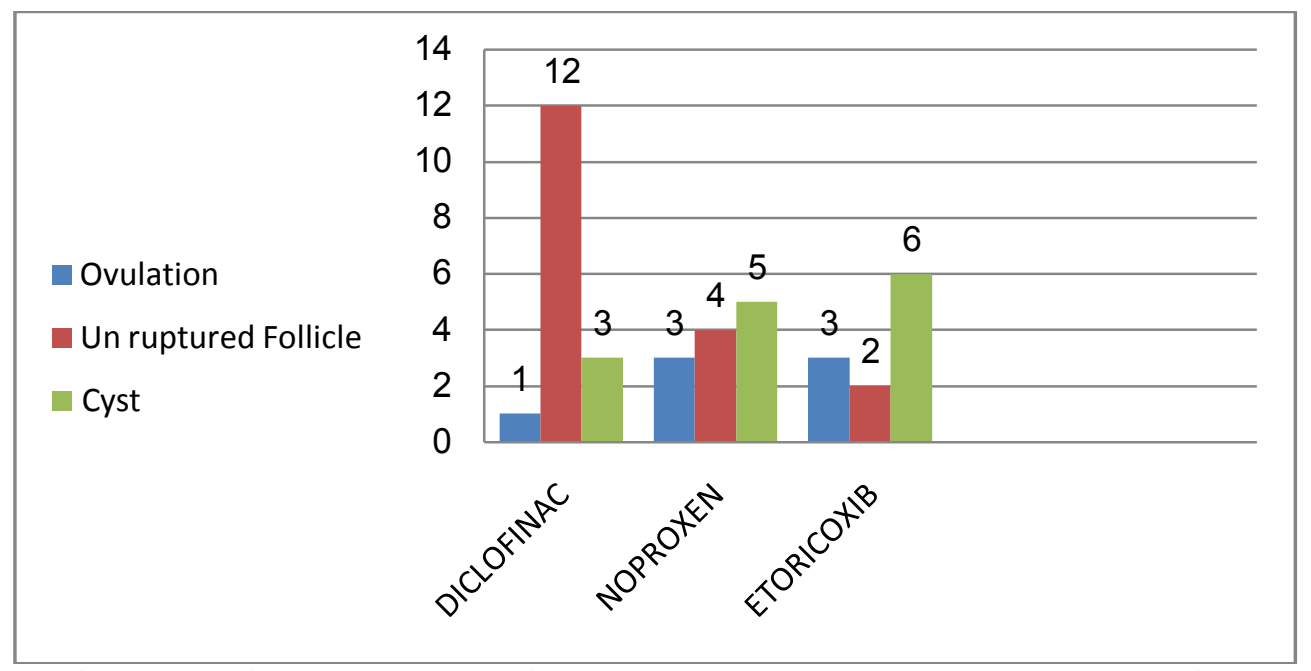

Fig 4: Fate of dominant follicle at the end of the treatment period ( day 20-22) with diclofenac, naproxen $\&$ etoricoxib

\section{Discussion}

Effects of Diclofenac, Naproxen \& Etoricoxib on dominant follicle ( DF) during Luteal phase:

Diclofenac, naproxen \& etoricoxib exhibited a highly inhibitory effects on ovulation compared to the control, demonstrated by growing follicle without ovulation during the treatment period, this is because PGs $\left(\mathrm{PGE}_{2} \& \mathrm{PGI}_{2}\right)$ are formed in preovulatory follicles in response to the preovulatory LH surge, and reach their highest concentrations around the time of ovulation (6), therefore treatment with either classical NSAIDs or selective COX-2 inhibitors (7), inhibit both PG synthesis and ovulation $(8,9,3)$, which can be restored (at least in some experimental conditions) by exogenous PG administration (10).

Moreover $\mathrm{PGF}_{2 \alpha}$ is a well known factor in terminating inflammatory changes associated with ovulation, this suggests that inhibition of $\mathrm{PGF}_{2 \alpha}$ by NSAIDs administration may block or prevent the termination of the inflammatory process and allowing it to extend longer than normal (11).

\section{Effects of Diclofenac, Naproxen \& Etoricoxib on progesterone level $\left(\mathrm{P}_{4}\right)$ during Luteal phase:}

It has been observed that diclofenac, naproxen \& etoricoxib caused a highly significant decrease in the mean $\mathrm{P}_{4}$ level, in comparison with the control during luteal phase, this results are consistent with other study have been previously done on human females, when compare meloxicam-treated group to placebo, a 5-day delay in follicle rupture, a $55.7 \%$ increase in the mean maximum follicle diameter, and $33.5 \%$ decrease of plasma progesterone level were observed. Concluded that meloxicam resulted in a reversible delay of ovulation, an increase in follicular diameter, and a decrease in plasma progesterone level (12). But regarding progesterone level before \& after treatment with either diclofenac, naproxen or etoricoxib, it has been observed statistical increased in $\mathrm{P}_{4}$ level during the luteal phase, this increment in progesterone level is below control level and this can give an indication for delayed ovulation because we took the blood samples at day 20-22 of the normal cycle (assuming 28 day cycle) while the dominant follicle still in the ovary for most patients that have been checked by US , and it's important to realize that if ovulation do not occur until day 16 ,so having the test on day 21 would be too early. It should really be taken 7 days post ovulation, which for convenience is called day 21 , that is for an average 28 day cycle (13).

\section{Effects of Diclofenac, Naproxen \& Etoricoxib on E.TH during luteal phase:}

Regarding effects on E.TH., after treatment with either diclofenac, naproxen or etoricoxib, normal increase in the E.TH. have been observed in comparing to control during the luteal phase, i.e. normal physiological events. Although COX-2 play important role in endometrial function (14), there are other factors affecting E.TH. other than COX enzymes which include patients age, it has been found that the mean endometrial thickness decreased as a function of the patient's age, the thickest endometrium was found in patients $<25$ years of age $(11.9 \pm 2.5 \mathrm{~mm})$, and the thinnest endometrium was found in patients $>40$ years of age $(9.6 \pm 2.3 \mathrm{~mm})$, and since most of our patients were $<30$ years old so their E.TH. considered within normal, also there is Estradiol $\left(\mathrm{E}_{2)}\right.$ levels which play a central role in the thickening of the endometrium (15), this $\mathrm{E}_{2}$ increased in response to LH surge prior to ovulation, and since $\mathrm{E}_{2}$ level did not affected by NSAIDs (16), so normal E.TH. were observed. 
These differences observed among the three groups of NSAIDs in their actions on these three parameters (DF, $\mathrm{P}_{4}$ level \& E.TH.) may reflect the differences in potency of anti-inflammatory action between the different groups of NSAIDs according to differences in COX enzyme selectivity \& in pharmacokinetic properties (17).

\section{Percentage reduction in ovulation by diclofenac, naproxen $\&$ etoricoxib:}

When diclofenac, naproxen \& etoricoxib, were given in a therapeutic dose during the late follicular phase, ovulation observed in low percentage While Unruptured follicles and cysts which reflect reduction in ovulatory process were observed with high percentage in diclofenac \& etoricoxib respectively.Diclofenac show the highest percentage of reduction in ovulatory process, because it show low to moderate preference to block the COX2-isoenzyme, approximately 10-fold (18), also larger sample size comparison to naproxen \& etoricoxib, in addition to the diclofenac dosage form which is sustained release preparation that help to increase patients compliance, all these factors may contribute to high percentage of ovulatory reduction .From another point of view etoricoxib which is preferential COX-2 inhibition with a long half-life, theoretically, were affected follicle rupture more often than nonselective NSAIDs or NSAIDs with a short half-life, since COX-2 seems to be of greater importance than COX-1 for the process of ovulation, $(19,20)$, but in this study show the lowest percentage of reduction in ovulation and this is can be explained by: small sample size and dose dependency for inhibition of follicle rupture. A dose dependency of ovulation inhibition was shown in a double-blind crossover study of a healthy women exposed to either $15 \mathrm{mg} /$ day or $30 \mathrm{mg} /$ day of meloxicam in the late follicular phase (21). Dysfunctional ovulation was observed in half of the patients treated with lower dose and ninety percent in the patients treated with higher doses of meloxicam (21). Also in another study complete inhibition of ovulation has been achieved by 150-200 $\mathrm{mg}$ of indomethacin (22). This indicates that prostanoids must be reduced below a certain threshold to result in LUF syndrome. this may explain why diclofenac, induced the luteinized Unruptured follicle (LUF) syndrome more frequently than naproxen \& etoricoxib.

moreover, nonselective NSAIDs and those with a shorter half-life like naproxen may allow proper COX production in drug-free intervals especially Patients who need to take a drug several times a day are often not compliant and take less than was prescribed.

\section{Fate of dominant follicle at the end of the treatment period ( day 20-22) with diclofenac, naproxen $\&$ etoricoxib:}

The administration of NSAIDs hinders completion of the ovulatory process which has been turned into luteinized Unruptured follicles (23), leading to the failure of the follicular rupture and hence formation of cysts (24),

In this study, it has been observed that the Unruptured follicles occur with the highest percentage for diclofenac group, decrease the percentage for naproxen group \& the lowest for etoricoxib group.

While cyst occur in the lowest percentage for diclofenac, increased for naproxen \&with the highest percentage for etoricoxib, but the overall percentage reduction in ovulation are statistically significant.

The differences among these three drugs in producing whether Unruptured follicles or cysts were time controlling, i.e. cyst consider as the fate for Unruptured follicle, because after ovulation, follicle collapses, but in cases of LUFS the follicle does not collapse and forms a cyst (25), and in case of etoricoxib most of patients were on day twelve of the cycle when began the treatment period for ten days, so the follow up have been done on day twenty two- twenty three of the cycle while the follow up for most patients treated with diclofenac \& naproxen have been done on day nineteen - twenty of the cycle.

At the same time in most cases, the cyst will naturally reabsorb itself (this has been observed during the follow up for the next cycle).

The results of this study confirm that the administration of diclofenac, naproxen \& etoricoxib respectively, during the late follicular phase (day 10-12) can interfere with the ovulatory process, either inhibit follicular rupture or delayed ovulation. Significant differences between these drugs in the outcome of the dominant follicle, and in the endocrine profile ( progesterone level) were observed, but no differences were found in other biological variables, such as cycle length.

\section{Conclusion}

a. Significant delay in ovulation have been observed in all treated groups between (93.75-72.7\%) .

b. Significant decrease in progesterone level below normal during luteal phase for the three drugs .

c. Cysts have been observed in about $30 \%$ of patients during the treatment with either diclofenac, naproxen or etoricoxib, were considered as fate of Unruptured follicles, which on following up for the next cycle have been disappeared. 


\section{References}

[1]. Stuart J. Warden (2010): "Prophylactic Use of NSAIDs by Athletes: A Risk/Benefit Assessment". The Physician and Sports Medicine; 38 (1): 132-138.

[2]. Espey LL.(1980): Ovulation as an inflammatory response - a hypothesis. Biol Reprod ;22:73-106.

[3]. Espey LL and Lipner H. (1994): Ovulation. In Knobil E and Neill JD (eds) Physiology of Reproduction;vol 1. Raven Press, New York: pp 725-781.

[4]. Armstrong DT(1981): Prostaglandins and follicular functions. J Reprod Fertil ;62:283-291.

[5]. Liu J and Sirois J.(1998): Follicle size-dependent induction of prostaglandin G/H synthase-2 during super ovulation in cattle. Biol Reprod ;58: 1527-1532.

[6]. Hedin L., Gaddy-Kurten G., Kurten R., Dewitt W. and Richards J. (1987): Prostaglandin endoperoxide synthase in rat ovarian follicles: content, cellular distribution and evidence for hormonal induction` preceding ovulation. Endocrinology;121: $722-731$.

[7]. Cryer B. and Dubois A (1998): The advent of highly selective inhibitors of cyclooxygenase- a review. Prostagland. \& Other Lipid Mediat; 56: 341-361.

[8]. Brännström M. and Janson P.O.(1991): The biochemistry of ovulation. In: Ovarian endocrinology. Hillier S.G. (ed). Blackwell Scientific Publications.Oxford; 132-166.

[9]. Tsafriri A., Chun S.Y. and Reich R.(1993): Follicular rupture and ovulation. In: The ovary. Adashi E.Y. and Leung P.C.K (eds). Raven Press. New York; 228-243.

[10]. Gaytán F., Tarradas E., Bellido C., Morales C. and Sánchez-Criado J.E. (2002a): Prostaglandin E1 inhibits abnormal follicle rupture and restores ovulation in indomethacin-treated rats. Biol. Reprod; 67: 1140-1147.

[11]. Murdoch WJ; CavenderJ.L. (1989): Effects of indo methacin on vascular architecture of pe-ovulatory ovin follicles possible implication in the lutenized unruptured follicle syndrome fert-steril;51(1):153-155.

[12]. Bata MS, Al-Ramahi M, Salhab AS, Gharaibeh MN, Schwartz J(2006): Delay of ovulation by meloxicam in healthy cycling volunteers: a placebo controlled, double blind, crossover study. J Clin Pharmacol;46:925-932.

[13]. Hambridge HL, Mumford SL, Mattison DR, Ye A, Pollack AZ, Bloom MS, Mendola P, Lynch KL, Wactawski-Wende J, Schisterman EF. (2013): Hum Reprod.;28(6):1687-94.

[14]. Jabbour HN, Kelly RW, Fraser HM, Critchely HO.(2006): Endocrine regulation of menstruation. Endocr.Rev; 27(1): P17-46.

[15]. Wiser Amir, Baum Micha, Hourwitz Ariel, Lerner-Geva Liat, Dor Jehoshua, .Shulman Adrian.(2007): Predicting factors for endometrial thickness during treatment with assisted reproductive technology. Fertility and Sterility; 87(4):799-804.

[16]. Al_atrakji M. Q., Al-Zoharyi A. M. \& Al-Janabi A. S. (2012): Comparative study of the effects of some NSAIDs on ovulation in female mice . Journal of the Faculty of Medicine- Baghdad; 54(2):158-162.

[17]. Vane JR \& Botting RM.(1998): Anti-inflammatory drugs and their mechanism of action. Inflammation Research; 4 S:78-S87.

[18]. Dutta NK, Annadurai S, Mazumdar K, Dastidar SG, Kristiansen JE, Molnar J, Martins M, Amaral L (2000): "The anti-bacterial action of diclofenac shown by inhibition of DNA synthesis". Int. J. Antimicrob. Agents;14 (3): $249-51$.

[19]. Mikuni M, Pall M, Peterson CM, Peterson CA, Hellberg P, Brannstrom M, Richards JS and Hedin L.(1998): The selective prostaglandin endoperoxide synthase-2 inhibitor, NS-398, reduces prostaglandin production and ovulation in vivo and in vitro in the rat. Biol Reprod; 59:1077-1083.

[20]. Richards JS (2001):Perspective: the ovarian follicle. Endocrinology;142:2184-93.

[21]. Jesam C, Salvatierra AM, Schwartz JL, Croxatto HB. (2010): Suppression of follicular rupture with meloxicam, a cyclooxygenase-2 inhibitor: potential for emergency contraception. Hum Reprod; 25(2):368-73.

[22]. Akil M, Amos RS, Stewart P(1996): Infertility may sometimes be associated with NSAID consumption. Br J Rheumatol;35:76-8.

[23]. Priddy AR ; Killick SR; Elisten-M; Morris-J; Sullivan-M; Patek-L;Elder-M.(1990): The effect of prostaglandin synthase inhibitors on human pre-ovulatory follicular fluid prostaglandin,thromboxane and leukotrines concentration J.Clin.Endocrinology.Metab;71(1):235-42.

[24]. Silva M.D \& Reeves J.J.(1985): Indomethacin inhibition of ovulation in the cow.J.Reprod.fert.;75:547-54.

[25]. Ashby, Karen L. (2004): "Ovarian Cyst". In Loue, Sana \& Sajatovic, Martha. Encyclopedia of Women's Health. Springer , p. 476. ISBN 9780306480737. 